\title{
Combination electrochemotherapy: another solution for triple negative breast cancers
}

\begin{abstract}
Triple negative breast cancer (TNBC) has a poor prognosis. This is associated with a lack of estrogen, progesterone and HER-2 receptors. About $15 \%$ of all diagnosed breast cancers are categorized as TNBC. This year, in the US alone, over 10000 women will die of TNBC. This grade of breast cancer is very aggressive and currently has very limited treatment options because standard endocrine therapies are largely ineffective. Hence, finding viable treatment options is an important area of research. Considering that the current standard of care does not serve all patients, there is a critical need for the development of alternate therapies such as electrochemotherapy (ECT), where using electrical pulses for enhanced uptake of chemotherapy drugs has the potential to improve TNBC patient outcomes. In ECT, electrical pulses are applied to transiently disrupt cell memebrane integrity to facilitate the entry of chemotherapy drug molecules that are usually impermeable. Typically Bleomycin (occasionally Cisplatin) is used in conjunction with ECT. In clinical practice for TNBC, a combination of Gemcitabine and Cisplatin is used in light of their elevated effectiveness, compared to use of a single drug. Hence, in this study we explore the efficacy of the Gemcitabine+Cisplatin combination in conjuction with ECT on the viability of MDA-MB-231, triple negative, human breast cancer cells. In brief, our results indicate that the coupling of ECT with chemotherapy drugs leads to increased mammary tumor cell death, as compared to use of drugs without ECT. These studies suggest ECT combination therapy may have the potential to enhance the efficacy of standard TNBC treatments and that further studies are necessary towards the development of this technique for possible clinical utility.
\end{abstract}

Volume 3 Issue 6 - 2015

Raji Sundararajan,' Raman V, ${ }^{2}$ Camarillo IG'
'School of Engineering Technology, Purdue University, USA
${ }^{2}$ Department of Biological Sciences, Purdue University, USA

Correspondence: Raji Sundararajan, Purdue University 40I, N Grant St, West Lafayette, IN 47907, USA, Tel I 765494 69I2, Email raji@purdue.edu

Received: October 30, 2015 | Published: December 07, 2015

\section{Background}

Triple negative breast cancers (TNBC) cell lack the genes for estrogen receptor (ER), progesterone receptor (PR) and human epidermal growth factor receptor 2 (HER $2 / n e u),{ }^{1}$ and are therefore resistant to standard endocrine therapies resist. Thus, TNBC carries an extremely poor prognosis, and are usually very aggressive. The high proliferative index, of TNBC makes it susceptible to chemotherapy. However, with inherent DNA repair defects and high occurrence of visceral metastasis, there is a much too common high rate of rapid relapse with this disease. ${ }^{2}$ TNBC can occur at a young age and has a strong correlation with the patient's race. Figure 1 shows a comparison of the incidence and death rate of TNBC in white and black women. ${ }^{3}$ In general, TNBC patients have aggressive clinical course and adverse outcomes, compared to non-TNBC. In a recent study (Figure 2), that included a follow-up period of 8 years, $8.2 \%$ of non-TNBC patients died, as compared to $16.2 \%$ of TNBC patients (twice that of non-TNBC). ${ }^{4}$ Thus, there is a pressing need for safe, alternative, physical, effective, yet affordable treatments to improve the condition of TNBC patents. Towards this, this study addresses the hypothesis that electrical pulses could be used to enhance intracellular delivery and effects of chemotherapeutics in TNBC breast cancer cells and tumors, at very low doses. Also, using a combination of drugs, as in clinical practices, ${ }^{5-7}$ it is possible to overcome drug resistance partly due to summative/synergistic effect. We and others have shown that electrical pulses can drive large therapeutic molecules into cells, both in vitro, and in vivo. ${ }^{8-10}$ Supporting this, human clinical trials for skin cancers and chest wall breast carcinoma ${ }^{9,10}$ have demonstrated the efficacy of this technique, requiring only small dosages of chemo drug compared to conventional chemotherapy, thus minimizing side effects and reducing cost significantly. Especially, this treatment is ideally suited for inoperable, recurrent, advanced tumors.

In this research, the efficacy of two potent chemo drugs, Gemcitabine $(\mathrm{G})+$ Cisplatin (C) combination therapy is explored under various pulse and dose parameters. This combination is chosen as it is being practiced as both first-line and 2nd-line therapy for TNBC.

Cisplatin is a potent platinum-based chemo drug, used for a number of solid tumors, including head and neck, lung, ovarian, breast and brain, to mention just a few. It kills cancer cells by damaging DNA, inhibiting DNA synthesis and mitosis, and inducing apoptosis. ${ }^{5}$ Several molecular mechanisms of action occur including oxidative stess induction, induction of p53 signalling and cell cycle arrest, activation of both extrinsic and intrinsic pathways of apoptosis. However, cisplation chemotherapy is associated with a number of side effects, such as hepatotoxic, nephrotoxic, cardiotoxic, neurotoxic and/or hematotoxic damage. In addition, some patients may relapse with drug resistance. Hence combination therapies with other drugs are common. Typically, two drugs with different mechanisms of action are used, so that each cannot cancel the other effect if not synergistic. Typically, summative and/or synergistic effects are expected in combination drug therapy. Gemcitabine inhibits DNA polymerase $\alpha$. It is incorporated into DNA, terminating DNA-chain elongation. It has several resistance mechanisms which are not fully understood. Combination of Cisplatin, a platinum-based cytotoxic drug with gemcitabine is used as both 1 st line and 2nd line regimen for TNBC. ${ }^{6,11,12}$ Extending this combination application using electrical pulses, as electrochemotherapy is outpatient-based, safe, efficient, economical, with minimal side effects, has the potential to be transferred to clinical practice. 


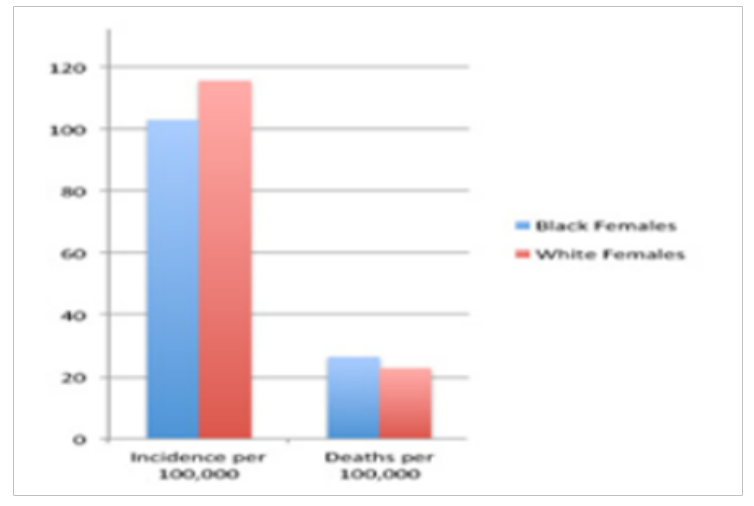

Figure I Comparison of Incidence and death rate ofWhite and Black women. ${ }^{3}$

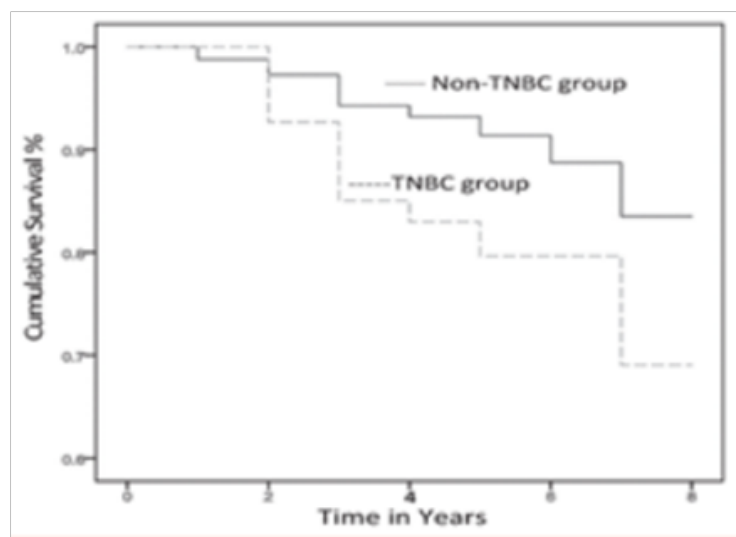

Figure 2 Comparison of TNBC and non-TNBC patients' \% cumulative survival. ${ }^{4}$

\section{Materials \& methods}

\section{The cells}

MDA-MB-231, human, adenocarcinoma, epithelial breast cancer cells, with ER, PR, and HER2 negative were used. They partially respond to chemotherapy.

\section{The drugs}

Gemcitabine (Sigma G6423) and Cisplatin (Sigma PHR1624) were used. $\mathrm{G}$ was solubilized in water at $10 \mathrm{mg} / \mathrm{mL}$ and concentrations of $100-400 \mu \mathrm{M}$ were used. C, solubilized in DMSO was also prepared at the same doses and used.Gemcitabine $\mathrm{HCl}$ is a $2^{\prime}$-deoxy- $2^{\prime}, 2^{\prime}$ difluorocytidine monohydrochloride ( $\beta$-isomer). Its chemical formula is $\mathrm{C} 9 \mathrm{H} 11 \mathrm{~F} 2 \mathrm{~N} 3 \mathrm{O} 4 \cdot \mathrm{HCl}$ and its structure is shown in Figure $3 \mathrm{~A} .{ }^{13}$ It is a nucleoside metabolic inhibitor. It replaces cytidine nucleic acid during DNA replication, arresting tumor growth, resulting in apoptosis. It is mainly used in pancreatic cancer. It is also used in bladder cancer, non-small cell lung cancer, and breast cancer, softtissue sarcoma and ovarian cancer. The various side effects include, easy bruising or bleeding, unusual weakness; urinating less than usual or not at all; nausea, upper stomach pain, itching, loss of appetite, dark urine, clay-colored stools, jaundice; chest pain. Cisplatin ((sp-4-2)diamminedichloridoplatinum) is a potent, platinum-based compound, in use since the mid-1800s. Its chemical structure is shown in Figure $3 b .{ }^{5}$ It binds to the genomic DNA (gDNA) in the cell nucleus and implements antitumor properties.

\section{The electroporation technique}

A BTX ECM 830 square wave electroporator (Genetronics, Inc., San Diego, CA) was used for the experiments. The various intensities studied are shown in Table 1 . The pulses were applied at $1 \mathrm{~Hz}$ (one second interval). These pulses were selected based on previous research. $^{8-10}$

\section{The viability assay}

$20 \mu \mathrm{L}$ of treated samples from cuvettes were added to counting chambers and were counted and the viability measured using a Nexcelom Bioscience Cellometer.

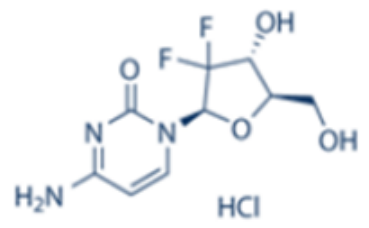

a

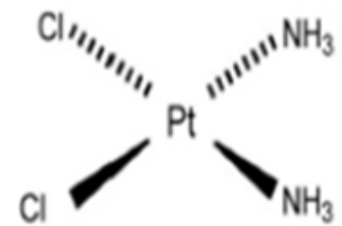

b
Figure 3A Chemical Structure of Gemcitabine.

Figure 3B Chemical Structure of Cisplatin. ${ }^{5,13}$

Table I Pulse conditions studied

\begin{tabular}{llll}
\hline Item & Intensity V/cm & Pulse duration & \# Pulses \\
\hline $\mathrm{I}$ & 1500 & $100 \mu \mathrm{s}$ & 8 \\
2 & 500 & $10 \mathrm{~ms}$ & 8 \\
3 & 500 & $25 \mathrm{~ms}$ & 2 \\
\hline
\end{tabular}

\section{Results and discussion}

Figure 4 shows the viability results for $\mathrm{G}+\mathrm{C}$ combination at 200 , 300 , and $400 \mu \mathrm{M}$ concentrations of each drug, when electroporated using $500 \mathrm{~V} / \mathrm{cm}, 10 \mathrm{~ms}, 8$ pulses. These were measured 90 minutes after pulsing. At $400 \mu \mathrm{M}$, the cell death is $56 \%$, corresponding to the viability of $44 \%$, while the cell deaths are $37 \%$ and $31 \%$ at 300 and $200 \mu \mathrm{M}$ respectively. The viability decreases with increase in dosage. This indicates that the $500 \mathrm{~V} / \mathrm{cm}$ pulse conditions are capable of reducing the proliferation of the MDA-MB-231 cells, achieving the desired outcome. Figure 5 shows a comparison of viabilities at two pulse conditions with the same $500 \mathrm{~V} / \mathrm{cm}$ intensity, but different pulse durations. Sample 1 was electroporated under $500 \mathrm{~V} / \mathrm{cm}, 2$ pulses at $25 \mathrm{~ms}$ pulse duration and Sample 2 at $500 \mathrm{~V} / \mathrm{cm}, 8$ pulses at $10 \mathrm{~ms}$ duration. The effect of longer pulse duration, though with only 2 pulses is seen in the case of sample 1 . Even though eight $10 \mathrm{~ms}$ pulses were applied for sample 2, the cell death is lower. This could be due to the high energy of individual pulses applied to sample 1 compared to sample 2. Figure 6 shows the viabilities at $100 \mu \mathrm{M}$ for $1500 \mathrm{~V} / \mathrm{cm}, 100 \mu \mathrm{s}, 8$ pulses and $500 \mathrm{~V} / \mathrm{cm}, 25 \mathrm{~ms}, 2$ pulses and the drug only viabilities for the three cases of $\mathrm{G}+\mathrm{C}$ only, $\mathrm{G}$ only and $\mathrm{C}$ only. At $1500 \mathrm{~V} / \mathrm{cm}, \mathrm{G}$ only has the lowest viability compared to $\mathrm{G}+\mathrm{C}$ and $\mathrm{C}$ only. However, at $500 \mathrm{~V} / \mathrm{cm}, \mathrm{G}+\mathrm{C}$ combination has highest cell death than $\mathrm{G}$ only and $\mathrm{C}$ only conditions.

In this concentration, there is only $15 \%$ cell death at $1500 \mathrm{~V} / \mathrm{cm}$ for $\mathrm{G}+\mathrm{C}$ combination. It was $26 \%$ for the $500 \mathrm{~V} / \mathrm{cm}$ pulses. The viability 
of $\mathrm{C}$ only is $30 \%$ higher than the $\mathrm{G}+\mathrm{C}$ combination, illustrating the higher efficacy of the combination. The $\mathrm{G}+\mathrm{C}$ condition could be used for clinical applications for effectively reducing the tumor size or cell proliferation. Table 2 shows the drug only, pulse only and control (with no treatment) viabilities, indicating the influence of the chemo drug + electrical pulse combination in obtaining the required cell kill to treat the tumor burden. With poor prognosis due to their molecular make-up, survival time for TNBC patients from distance recurrence to death is 9 months. ${ }^{14}$ There is an urgent clinical need for alternative therapies and ECT is a viable option. Since our body cells and tissues could elicit both therapeutic and diagnostic phenomena at various magnitudes and frequencies of electrical pulses, ECT is uniquely qualified to treat advanced, inoperable and chemoresistant TNBC tumors. Due to the synergy and/or summative effect of these two drugs, Gemcitabine + Cisplation combination is useful in providing survival advantage without the addition of notable toxicity. The combination is appropriate for treatment of patients with advanced tumors. ${ }^{5}$

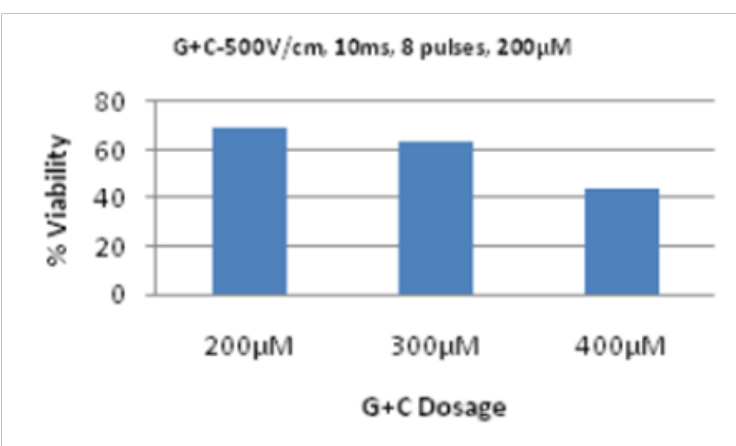

Figure $4 \mathrm{G}+\mathrm{C}$ at 200,300 and $400 \mu \mathrm{M}$ Concentrations at $500 \mathrm{~V} / \mathrm{cm}, 10 \mathrm{~ms}$ Pulse Conditions.

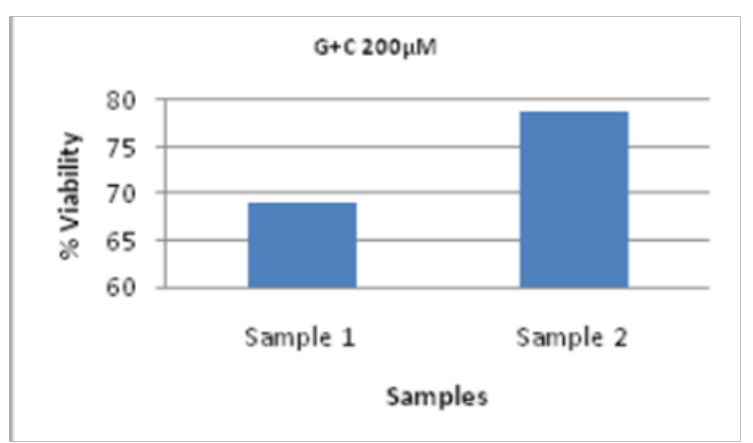

Figure 5 Comparison of $\mathrm{G}+\mathrm{C}$ at $200 \mu \mathrm{M}$ Concentrations at 500V, 25ms, 2 pulses (Sample I) and 500V/cm, I0ms, 8 pulses (Sample 2).

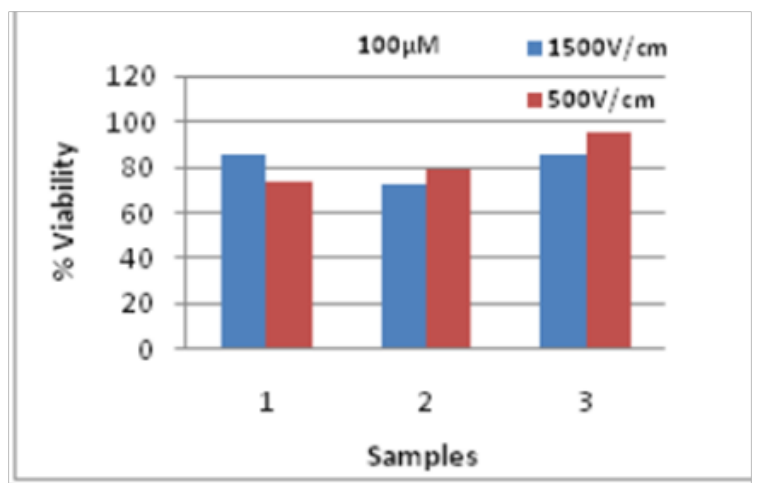

Figure 6 Viabilities at $1500 \mathrm{~V} / \mathrm{cm}, 100 \mu \mathrm{s}, 8$ pulses and $500 \mathrm{~V} / \mathrm{cm}, 25 \mathrm{~ms}, 2$ pulses-I) G+C, 2) G only and 3) C only.
Table 2 Viabilities at Various Conditions

\begin{tabular}{llll}
\hline Item & Sample & Dose $\boldsymbol{\mu M}$ & \% Viability \\
\hline I & G+C Only & 200 & 87.7 \\
2 & G Only & 200 & 90.6 \\
3 & C Only & 200 & 90.3 \\
4 & G Only & 100 & 91.2 \\
5 & Pulse Only & - & 92.5 \\
6 & Control & - & 95 \\
\hline
\end{tabular}

\section{Conclusion}

The combination of ECT with chemotherapy drugs $(\mathrm{G}+\mathrm{C} 400 \mu \mathrm{M})$ lead to a substantial reduction in tumor cell viability (44\%), compared to the treatment with chemotherapy drugs only ( $87 \%$ cell viability). This improvement of efficacy was also observed when ECT was combined with other dose levels of the $\mathrm{G}+\mathrm{C}$ drugs. This work suggests that optimizing the pulse conditions and drug dosages can lead to reduced tumor cell viability. Due to its physical nature the technique of electrochemotherapy, in combination with standard drugs may have strong potential to impact patient outcomes, after further groundwork is accomplished towards the transfer to clinical practice.

\section{Acknowledgements}

None.

\section{Conflicts of interest}

The authors declare that there is no conflict of interest.

\section{References}

1. Saha A, Chattopadhyay S, Azam M, et al. Clinical outcome and pattern of recurrence in patients with triple negative breast cancer as compared with non-triple negative breast cancer group. Clinical Cancer Investigation J. 2012;1(4):201-205.

2. Yardley D. Drug resistance and the role of combination chemotherapy in improving patient outcomes. Intl J of Breast Cancer. 2013:137414.

3. www. Google.com/blank.html, Oct 292015.

4. Hamm C, El-Masri M, Poliquin G, et al. A single-center chart review exploring the adjusted association between breast cancer phenotype and prognostics. Curr Oncol. 2011;18(4):191-196.

5. Dasari S, Tchounwou PB. Cisplatin in cancer therapy: molecular mechanisms of action. Eur J Pharmacol. 2014;740:364-378.

6. Ozkan M, Berk V, Kaplan MA, et al. Gemcitabine and Cisplatin combination chemotherapy in triple negative metastatic breast cancer previously treated with a Taxane/Antharacycline Chemotherapy: Multicenter Experience. Neoplasma. 2012;59(1):38-42.

7. Telli M. Optimizing chemotherapy in triple-negative breast cancer: the role of platinum. Am Soc Clin Oncol Educ Book. 2014:e37-e42.

8. Sundararajan R, Xiao F, Salameh T, et al. Effective proliferation control of human cancer cells using electrical pulses. Dielectrics and Electrical Insulation. 2012;19(6):2225-2236.

9. Gehl J, Geertsen PF. Efficient palliation of haemorrhaging malignant melanoma skin metastases by electrochemotherapy. Melanoma Research. 2000;10(6):585-589.

10. Larkin JO, Collins CG, Aarons S, et al. Electrochemotherapy: aspects of practical development and early clinical experience. An of Surger. 2007;245(3):469-479. 
11. Verma S, Provencher L, Dent R. Emerging trends in the treatment of triple-negative breast cancer in Canada: a survey. Curr Oncol. 2011;18(4):180-190.

12. Zhang J, Wang $\mathrm{Z}, \mathrm{Hu} \mathrm{X}$, et al. Cisplain and gemcitabibe as the first line therapy in metastaic triple negative breast cancer. Int $J$ Cancer. 2015;136(1):204-211.
13. www. Google.com/blank.html, Oct 292015.

14. Wu S, Guo J, Wei W, et al. Enhanced breast cancer therapy with nsPEFs and low concentrations of gemcitabine. Cancer Cell Int. 2014;14(1):98. 